\title{
ПАРАМЕТРИЧЕСКАЯ ОПТИМИЗАЦИЯ СИСТЕМНОГО РЕГУЛЯТОРА СОГЛАСОВАННОГО УПРАВЛЕНИЯ ЭЛЕКТРОПРИВОДАМИ ЧЕТЫРЁХКОЛЁСНОГО МОБИЛЬНОГО РОБОТА
}

\section{CONSTRUCTION OF A TRAJECTORY OF PROGRAMMED MOTION \\ FOR COORDINATED CONTROL SYSTEM \\ OF ELECTRIC DRIVES OF A FOUR-WHEEL MOBILE ROBOT}

E. Meshkovskiy

Summary. The problem of parametric optimization of the system controller of coordinated control of a four-wheeled mobile robot with two differential drive units is considered. In the process of solving it, the objective function was derived, a criterion was compiled, and a method for optimizing parameters was selected. In conclusion, the article presents the results of numerical experiments on a graph the trajectory errors before and after the parametric optimization of a nonlinear and a fuzzy system controller.

Keywords: wheel robot, differential drive unit, coordinated control, parametric optimization, batch learning, conjugate gradient method, Brent method.

\author{
Мешковский Евгений Олегович \\ Аспирант, Санкт-Петербургский политехнический \\ университет Петра Великого \\ meshkovskii_evge@mail.ru
}

Аннотация. Рассмотрена задача параметрической оптимизации системного регулятора согласованного управления четырёхколёсного мобильного робота с двумя дифференциальными приводными блоками. В процессе её решения была выведена целевая функция, составлен критерий и выбран метод оптимизации параметров. В заключении статьи приведены результаты численных экспериментов по построению графиков траекторной ошибки до и после параметрической оптимизации нелинейного и нечёткого системного регулятора.

Ключевые слова: колёсный робот, дифференциальный приводной блок, согласованное управление, параметрическая оптимизация, пакетное обучение, метод сопряжённых градиентов, комбинированный метод Брента.

можно описать как поиск среди параметров системы $k$ множества $X$ такого набора параметров $k^{*}$, что

$$
f\left(k^{*}\right)=\min _{k \in X} f(k),
$$

где $f($ ) - целевая функция (отображение $f: X \rightarrow R$ ).

Постановка задачи оптимизации начинается с определения множества допустимых параметров, целевой функции и критерии оптимизации. Наши системные регуляторы разработаны так, что все коэффициенты, входящие в их структуру, должны быть положительными вещественными числами:

$$
X=\left\{k \in \mathbb{R}^{n} \mid k_{i}>0\right\} .
$$

Основная задача, выполняемая колёсными роботами в технологическом процессе, заключается в перемещении вдоль траектории. Требованием при выполнении поставленной задачи является минимальное отклонение от заданного контура в процессе перемещения. По этой причине целевой функцией выбран квадрат среднеквадратичного отклонения (с.к.о.) [4, с. 3] от тра- 


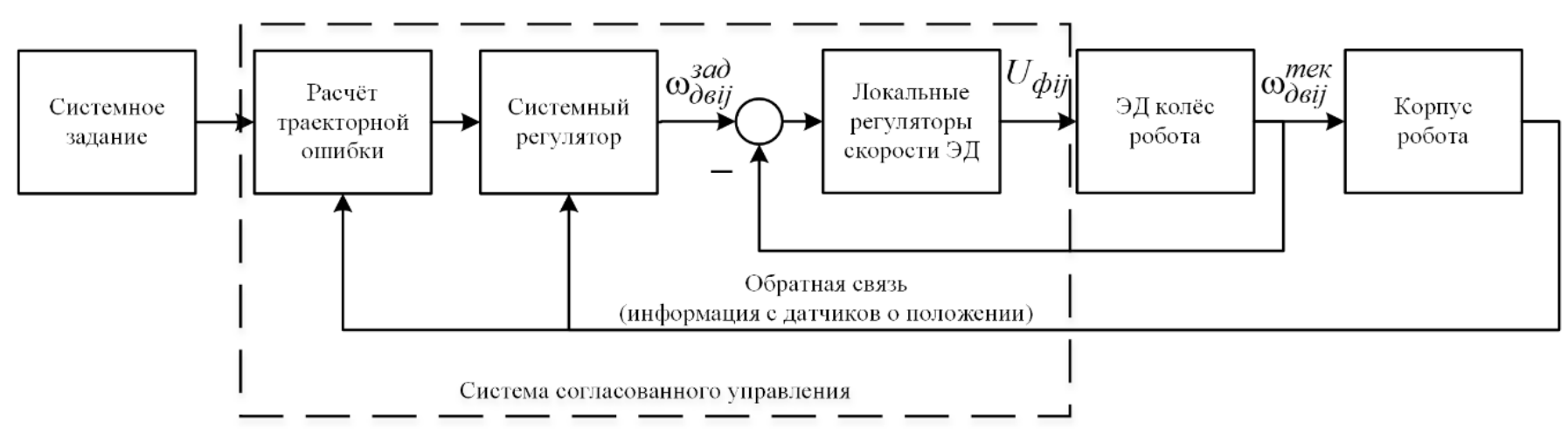

Рис. 1. Система согласованного управления электроприводами исследуемого колёсного робота

ектории (контурная ошибка), а критерием оптимизации - его минимум:

$$
\left\{\begin{array}{l}
f_{c}(k)=\varepsilon_{c}^{2}(k), \\
I_{c}=f_{c}(k) \rightarrow \min
\end{array}\right.
$$

где $\varepsilon_{c}^{2}(k)-$ квадрат с.к.о. контурной ошибки; $I_{c}-$ критерий оптимальности по контурной ошибке.

Кроме точности позиционирования относительно траектории существует также требование к минимальному угловому отклонению (угловой ошибке). Данный факт приводит нас к необходимости ввести дополнительную целевую функцию и критерий оптимизации, которые будут определяться квадратом с.к.о. углового позиционирования:

$$
\left\{\begin{array}{l}
f_{\beta}(k)=\varepsilon_{\beta}^{2}(k), \\
I_{\beta}=f_{\beta}(k) \rightarrow \min ,
\end{array}\right.
$$

где $\varepsilon_{\beta}^{2}(k)-$ квадрат с.к.о. ошибки углового позиционирования; $I_{\beta}$ - критерий оптимальности по угловому позиционированию.

В современном мире, с целью увеличения точности и скорости обучения нейронных сетей, используют пакетную (batch) оптимизацию [5, 6]. Суть данного способа заключается в обучении нейронной сети на всех обучающих выборках одновременно. Применим пакетную оптимизацию к нашей задаче параметрической оптимизации системных регуляторов, т.к. процесс обучения нейронной сети и оптимизация параметров наших элементов системы управления, по своей сути, процессы тождественные.

Пакеты формируются из наборов траекторий и заданных контурных скоростей. В итоге получаем набор из нескольких критериев (2) и (3), что приводит нас к задаче многокритериальной оптимизации:

$$
I_{D}=I_{D}\left(\varepsilon_{c 1}^{2}(k), \ldots, \varepsilon_{c n}^{2}(k), \varepsilon_{\beta 1}^{2}(k), \ldots, \varepsilon_{\beta n}^{2}(k)\right) \rightarrow \min , \text { (4) }
$$

где $I_{D}-$ итоговый «суперкритерий»; $\varepsilon_{c i}^{2}-$ квадрат с.к.о. контурной ошибки при $i$-ом испытании в пакете; $\varepsilon_{\beta i}^{2}-$ квадрат с.к.о. углового позиционирования при $i$-ом испытании; $n$ - количество элементов (испытаний) в одном пакете.

Решить поставленную задачу многокритериальной оптимизации нам поможет линейная свёртка критериев [7]:

$$
I_{D}=\sum_{i=1}^{n}\left(\alpha_{c i} \cdot \varepsilon_{c i}^{2}(k)+\alpha_{\beta i} \cdot \varepsilon_{\beta i}^{2}(k)\right) \rightarrow \min ,
$$

$\alpha_{c i}, \alpha_{\beta i}-$ вещественный коэффициент $i$-го критерия контурной ошибки и углового позиционирования соответственно. Указанные вещественные коэффициенты определяют степень важности соответствующего критерия, их сумма равна 1, т.е. весовые коэффициенты должны принадлежать множеству $A$ такому, что:

$$
\mathrm{A}=\left\{\alpha \in \mathbb{R}^{n} \mid \alpha_{c i}, \alpha_{\beta i}>0,\left(\sum_{i=1}^{n} \alpha_{c i}+\sum_{i=1}^{n} \alpha_{\beta i}\right)=1\right\} .
$$

Выражения (5) и (6) называют взвешенной суммой критериев или аддитивная форма свёртки критериев, которая применяется в задачах многокритериальной оптимизации $[8,9]$. Примем все

$$
\alpha_{c i}=\alpha_{\beta i}=\frac{1}{2 \cdot n}
$$

т.к. результаты всех испытаний в пакете, и по траекторной ошибке, и по угловому положению имеют для нас равную степень важности.

Докажем существования минимума гиперповерхности критерия (5). Для этого будем считать целевую функцию критерия (5) как функцию многих переменных 
от с.к.о. траекторной и угловой ошибки. Получим дважды дифференцируемую функцию:

$$
I_{D}=f\left(\varepsilon_{c 1}, \ldots, \varepsilon_{c n}, \varepsilon_{\beta 1}, \ldots, \varepsilon_{\beta n}\right)=\sum_{i=1}^{n}\left(\alpha_{c i} \cdot \varepsilon_{c i}^{2}+\alpha_{\beta i} \cdot \varepsilon_{\beta i}^{2}\right) .
$$

Необходимое условие существования локального экстремума гласит, что функция

$$
I_{D}=f\left(\varepsilon_{c 1}, \ldots, \varepsilon_{c n}, \varepsilon_{\beta 1}, \ldots, \varepsilon_{\beta n}\right)
$$

имеет в точке

$$
I_{D}^{*}\left(\varepsilon_{c 1}^{*}, \ldots, \varepsilon_{c n}^{*}, \varepsilon_{\beta 1}^{*}, \ldots, \varepsilon_{\beta n}^{*}\right)
$$

локальный экстремум если дифференциал в этой точке $d I_{D}\left(I_{D}^{*}\right)$ равен нулю или не существует $[10$, с. 364]. Возьмём частные производные по всем $\varepsilon_{c i}$ и $\varepsilon_{\beta i}$ от $I_{D}$ и приравняв их к нулю:

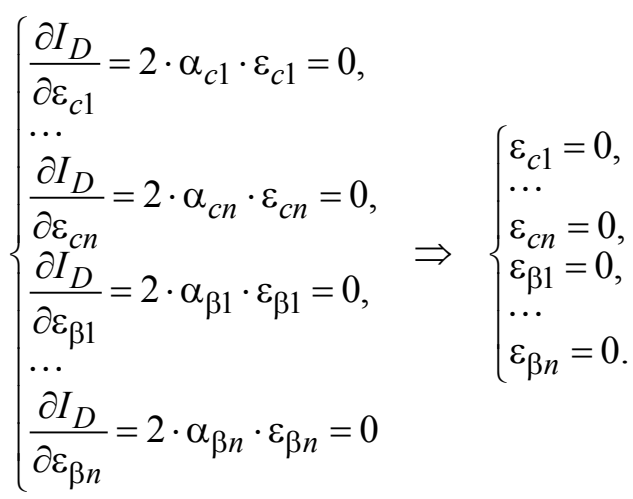

Результат в (8) даёт возможность предположить, что точка

$$
I_{D}^{*}(0, \ldots, 0,0, \ldots, 0)
$$

является локальным экстремумом. Данный факт необходимо подтвердить (точка $I_{D}^{*}$ может быть, к примеру, седловой) и определить, что $I_{D}^{*}$ является именно точкой минимума, а не максимума. С этим нам поможет достаточное условие существования локального экстремума, которое гласит, что если второй дифференциал

$$
d^{2} I_{D}\left(I_{D}^{*}\right)
$$

от всех переменных $d \varepsilon_{c i}$ и $d \varepsilon_{\beta i}$ в точке экстремума $I_{D}^{*}$ является положительно определённой формой, то функция

$$
I_{D}=f\left(\varepsilon_{c 1}, \ldots, \varepsilon_{c n}, \varepsilon_{\beta 1}, \ldots, \varepsilon_{\beta n}\right)
$$

имеет локальный минимум в точке $I_{D}^{*}[10$, с. 365].
Рассматриваемая функция (7) является одним из видов квадратичной формы [11, с. 196], Гессиан которой (матрица Гессе), в любой точке будет выглядеть как квадратная диагональная матрица с положительными элементами:

$$
\begin{aligned}
& H\left(I_{c}\right)= \\
& \left(\begin{array}{cccccc}
\frac{\partial^{2} I_{D}}{\partial \varepsilon_{c 1}^{2}} & \cdots & \frac{\partial^{2} I_{D}}{\partial \varepsilon_{c 1} \cdot \partial \varepsilon_{c n}} & \frac{\partial^{2} I_{D}}{\partial \varepsilon_{c 1} \cdot \partial \varepsilon_{\beta 1}} & \cdots & \frac{\partial^{2} I_{D}}{\partial \varepsilon_{c 1} \cdot \partial \varepsilon_{\beta n}} \\
\vdots & \ddots & \vdots & \vdots & \ddots & \vdots \\
\frac{\partial^{2} I_{D}}{\partial \varepsilon_{c n} \cdot \partial \varepsilon_{c 1}} & \cdots & \frac{\partial^{2} I_{D}}{\partial \varepsilon_{c n}^{2}} & \frac{\partial^{2} I_{D}}{\partial \varepsilon_{c n} \cdot \partial \varepsilon_{\beta 1}} & \cdots & \frac{\partial^{2} I_{D}}{\partial \varepsilon_{c n} \cdot \partial \varepsilon_{\beta n}} \\
\frac{\partial^{2} I_{D}}{\partial \varepsilon_{\beta 1} \cdot \partial \varepsilon_{c 1}} & \cdots & \frac{\partial^{2} I_{D}}{\partial \varepsilon_{\beta 1} \cdot \partial \varepsilon_{c n}} & \frac{\partial^{2} I_{D}}{\partial \varepsilon_{\beta 1}^{2}} & \cdots & \frac{\partial^{2} I_{D}}{\partial \varepsilon_{\beta 1} \cdot \partial \varepsilon_{\beta n}} \\
\vdots & \ddots & \vdots & \vdots & \ddots & \vdots \\
\frac{\partial^{2} I_{D}}{\partial \varepsilon_{\beta n} \cdot \partial \varepsilon_{c 1}} & \cdots & \frac{\partial^{2} I_{D}}{\partial \varepsilon_{\beta n} \cdot \partial \varepsilon_{c n}} & \frac{\partial^{2} I_{D}}{\partial \varepsilon_{\beta n} \cdot \partial \varepsilon_{\beta 1}} & \cdots & \frac{\partial^{2} I_{D}}{\partial \varepsilon_{\beta n}^{2}}
\end{array}\right)= \\
& =\left(\begin{array}{cccccc}
2 \cdot \alpha_{c 1} & \cdots & 0 & 0 & \cdots & 0 \\
\vdots & \ddots & \vdots & \vdots & \ddots & \vdots \\
0 & \cdots & 2 \cdot \alpha_{c n} & 0 & \cdots & 0 \\
0 & \cdots & 0 & 2 \cdot \alpha_{\beta 1} & \cdots & 0 \\
\vdots & \ddots & \vdots & \vdots & \ddots & \vdots \\
0 & \cdots & 0 & 0 & \cdots & 2 \cdot \alpha_{\beta n}
\end{array}\right)
\end{aligned}
$$

По критерию Сильвестра матрица (9) будет положительно определённой только при положительном значении всех её угловых миноров [11, с. 211]. Из (6) $\alpha_{c i}, \alpha_{\beta i}>0$

- Тогда, все миноры будут также строго больше нуля. Следовательно, (9) является положительно определённой, что в итоге приводит нас к выводу о наличии локального экстремума типа минимум функции (7) в точке

$$
I_{D}^{*}(0, \ldots, 0,0, \ldots, 0) .
$$

По этой причине критерий оптимизации (5) сходится к минимуму в

$$
I_{D}^{*}(0, \ldots, 0,0, \ldots, 0)
$$

и мы можем применить её для нашего исследования.

Заключительным этапом нашей задачи является определения метода оптимизации. Наиболее популярными методами оптимизации остаются градиентные алгоритмы первого порядка $[12,13]$, среди которых мы возьмём метод сопряжённых градиентов в форме Флетчера-Ривса [14, с. 67-68], который производит движение 


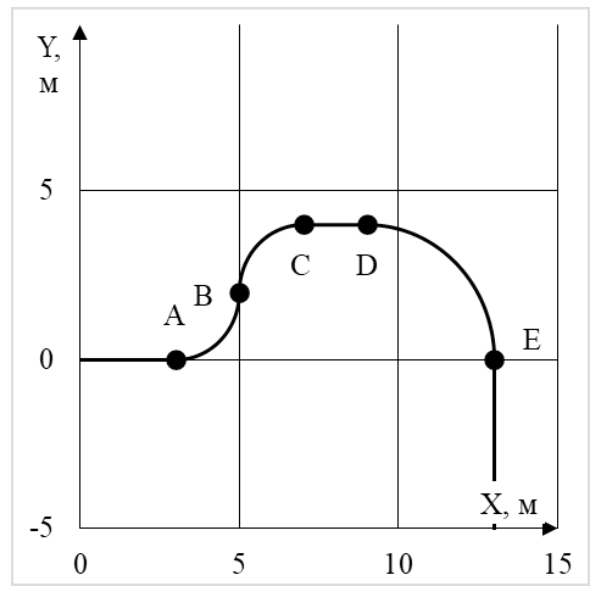

Рис. 2. Траектория для проведения параметрической оптимизации
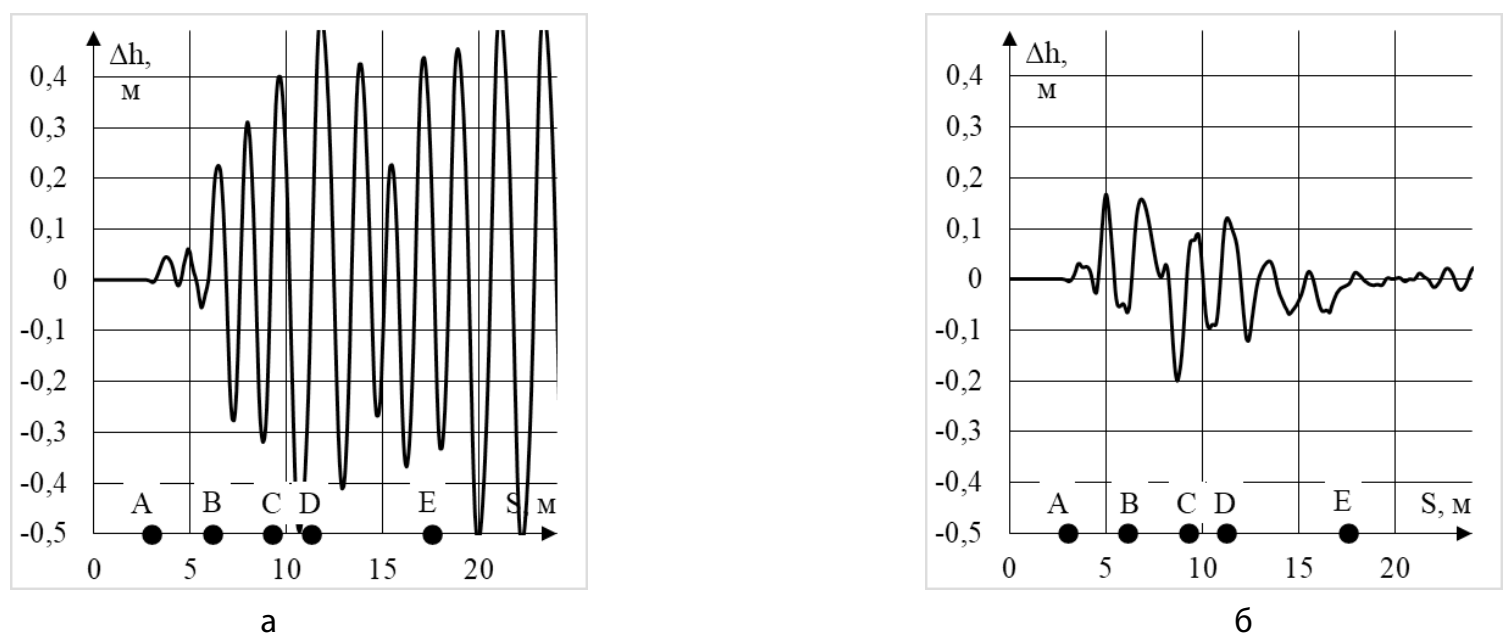

Рис. 3. Поперечное отклонение от траектории центра корпуса при использовании нелинейного системного регулятора: а — до оптимизации параметров; 6 - после оптимизации параметров

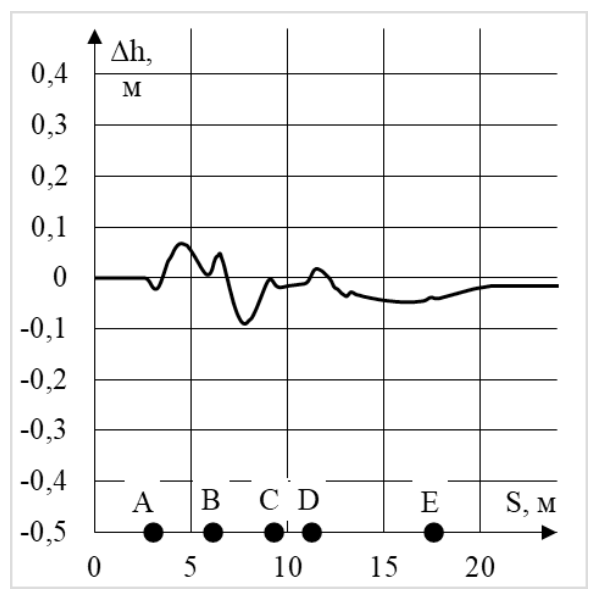

a

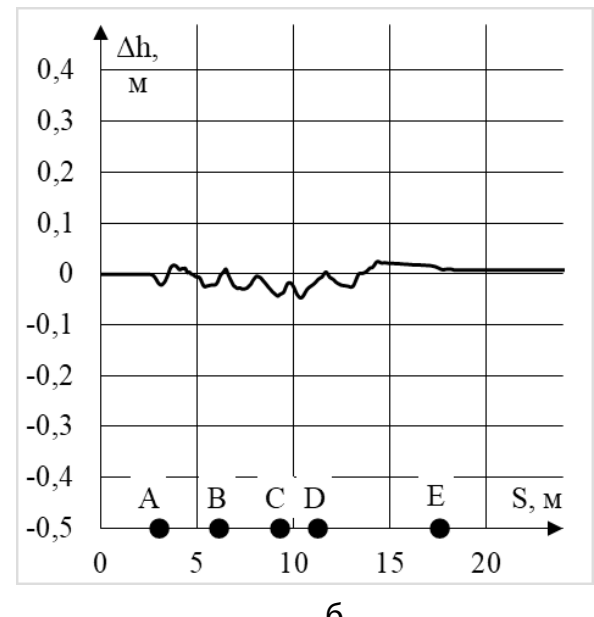

6

Рис. 4. Поперечное отклонение от траектории центра корпуса при использовании нечёткого системного регулятора: а - до оптимизации параметров; 6 - после оптимизации параметров 
к минимуму вдоль ортогонального направления к полученным на предыдущих шагах:

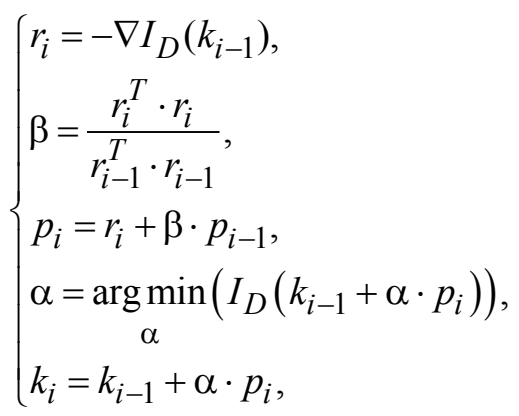

где $i$ - номер текущего шага оптимизации (поиска минимума); $k_{i}, k_{i-1}-$ значения параметров регуляторов на текущем и предыдущем шаге; $r_{i}, r_{i-1}$ - вектор антиградиента целевой функции на текущем и предыдущем шаге; $\beta$ - коэффициент сопряжения; $p_{i}, p_{i-1}-$ направление движения к минимуму на текущем и предыдущем шаге; $\alpha$ - величина шага в сторону минимума. Шага движения к минимуму $\alpha$ будем находить комбинированным методом Брента [15, с. 61-81], сочетающий метод парабол и золотого сечения.

Продемонстрируем применение метода (10) с критерием (5) при помощи численных экспериментов на модель исследуемого колёсного робота [16] массой 100 кг. Составим пакет из траектории (рис. 2) и набора из четырёх заданных контурных скоростей $v_{c}^{3 a d}: 0,25,0,50,0,75$ и 1,00 метров в секунду. На рис. 2 буквами $A, B, C, D$ и $E$ обозначены точки сопряжения траекторий. Проведём оптимизацию параметров нелинейного и нечёткого системного регулятора для продольно-симметричной конфигурации расположения приводных блоков

$$
\left(L_{1}=\left[\begin{array}{ll}
0.5, & 0.0
\end{array}\right]^{T}, L_{2}=\left[\begin{array}{ll}
-0.5, & 0.0
\end{array}\right]^{T},\right.
$$

в метрах). Максимальное число шагов оптимизации равно девяти. Локальные регуляторы скорости электродвигателей выбраны пропорционально-интегрирующего типа с настройкой на модульный (технический) оптимум [17].

После проведения параметрической оптимизации построим графики поперечного отклонения центра корпуса $\Delta h_{0}$ (в метрах) от пройденного пути $S$ (в метрах) при движении по траектории на расстояние 25 метров с контурной скоростью 1,00 метров в секунду. Рис. 3 демонстрирует результаты численных экспериментов, при управлении роботом нелинейным системным регулятором, рис. 4 - при использовании нечёткого системного регулятора.

Представленные результаты численных экспериментов для всех конфигураций расположения приводных блоков робота демонстрируют уменьшение контурной ошибки и стабильное движение. Поперечное отклонение не превышает 0,20 метра для нелинейного системного регулятора и 0,05 метра для нечёткого. Уменьшить данные показатели можно путём увеличения числа шагов и точности оптимизации.

В результате проведённого исследования был выведен критерий параметрической оптимизации системных регуляторов исследуемого четырёхколёсного мобильного робота с двумя дифференциальными приводными блоками. Полученная комбинация критерия и метода минимизации позволяет уменьшить контурную ошибку центра корпуса при использовании различной структуры системного регулятора. Дальнейшие исследования будут направлены на внедрение данного способа поиска параметров в промышленные колёсные.

\section{ЛИТЕРАТУРА}

1. Белоглазов, Д.А. Критерии функционирования систем автоматического управления / Д. А. Белоглазов, С. Е. Бублей // Известия ЮФу. Технические науки. - 2010.— № 7.—C. 185-191.

2. Грубый, С. В. Нелинейная оптимизация режимных параметров точения методом штрафной функции / С. В. Грубый // Известия вузов. Машиностроение.- 2018. — № 4 (697). - С. 3-9.

3. Дунаев, М. П. Параметрическая оптимизация системы управления насосной станцией с помощью генетического алгоритма / М. П. Дунаев, Н. Н. Куцый, Н. Д. Лукьянов // Наука и образование: научное издание МГТУ им. Н. э. Баумана. — 2014. — № 8. — C. 194-205.

4. ГОСТ Р 8.736-2011 Государственная система обеспечения единства измерений (ГСИ). Измерения прямые многократные. Методы обработки результатов измерений. Основные положения.- М: Стандартинформ, 2013. - 19 с.

5. Зуев, В. Н. Модифицированный алгоритм обучения нейронных сетей / В. Н. Зуев, В. К. Кемайкин // Программные продукты и системы.— 2019.— Т. 32, № 2.- C. 258-262.

6. Мелихова, О. А. Режимы обучения в искусственных нейронных сетях / О. А. Мелихова, О. В. Вепринцева, В. С. Чумичев, С. В. Джамбинов, А. Б. Гайдуков // Инновации в науке. - 2016. - № 50-1. - С. 16-23.

7. Меламед, И. И. Линейная свертка критериев в многокритериальной оптимизации / И.И. Меламед // Автоматика и телемеханика.— 1997.—№ 9.—C. 119-125.

8. Ногин, В. Д. Линейная свертка критериев в многокритериальной оптимизации / В. Д. Ногин // Искусственный интеллект и принятие решений. - 2014.№ 4.- - . 73-82. 
9. Подиновский, В. В. Метод взвешенной суммы критериев в анализе многокритериальных решений: Pro et contra / В. В. Подиновский, М. А. Потапов // Бизнес-информатика. - 2013. - № 3 (25).- - С. 41-48.

10. Хорошилова, Е. В. Высшая математика. Лекции и семинары: учебное пособие для академического бакалавриата.— М.: Издательство Юрайт.— 2019.— 452 c.

11. Шафаревич, И. Р. Линейная алгебра и геометрия / И. Р. Шафаревич, А. О. Ремизов. — М.: ФИЗМАТЛИТ. — 2009. — 512 с.

12. Запрягаев, С. А. Вычисление и обучение искусственных нейронных сетей прямого распространения на графическом процессоре / С. А. Запрягаев, А. А. Карпушин // Вестник ВГУ: Системный анализ и информационные технологии. — 2011. — № 1. - C. 157-164.

13. Скороходов, А. В. Сравнительный анализ градиентных методов минимизации в задаче обучения многослойного персептрона / А. В. Скороходов, А. В. Тунгусова // Доклады ТУСУРа. — 2011.—№ 2 (24).—C. 98-102.

14. Осовский, С. Нейронные сети для обработки информации / Пер. с польского И. Д. Рудинского.— М.: Финансы и статистика. - 2002.

15. Brent, R. P. Algorithms for Minimization Without Derivatives. — Mineola, New York: Dover Publication, Inc. — 2013.— 208 p.

16. Мешковский, Е.О. Построение математической модели четырёхколёсного мобильного робота с двумя дифференциальными приводными блоками / Е. 0. Мешковский, А. Д. Курмашев // Инновации и Инвестиции. — 2020.— № 2.—C. 113-118.

17. Кояин, Н. В. Оптимизация контуров регулирования систем электропривода по типовым методикам / Н. В. Кояин, О. П. Мальцева, Л. С. Удут // Известия Томского политехнического университета. Инжиниринг георесурсов.— 2005.— № 7.— С. 120-125.

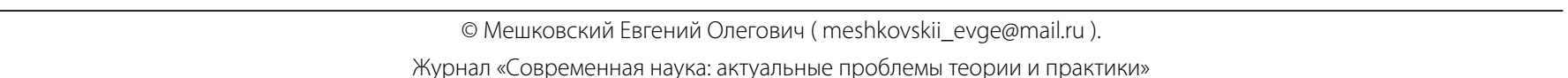

Журнал «Современная наука: актуальные проблемы теории и практики»

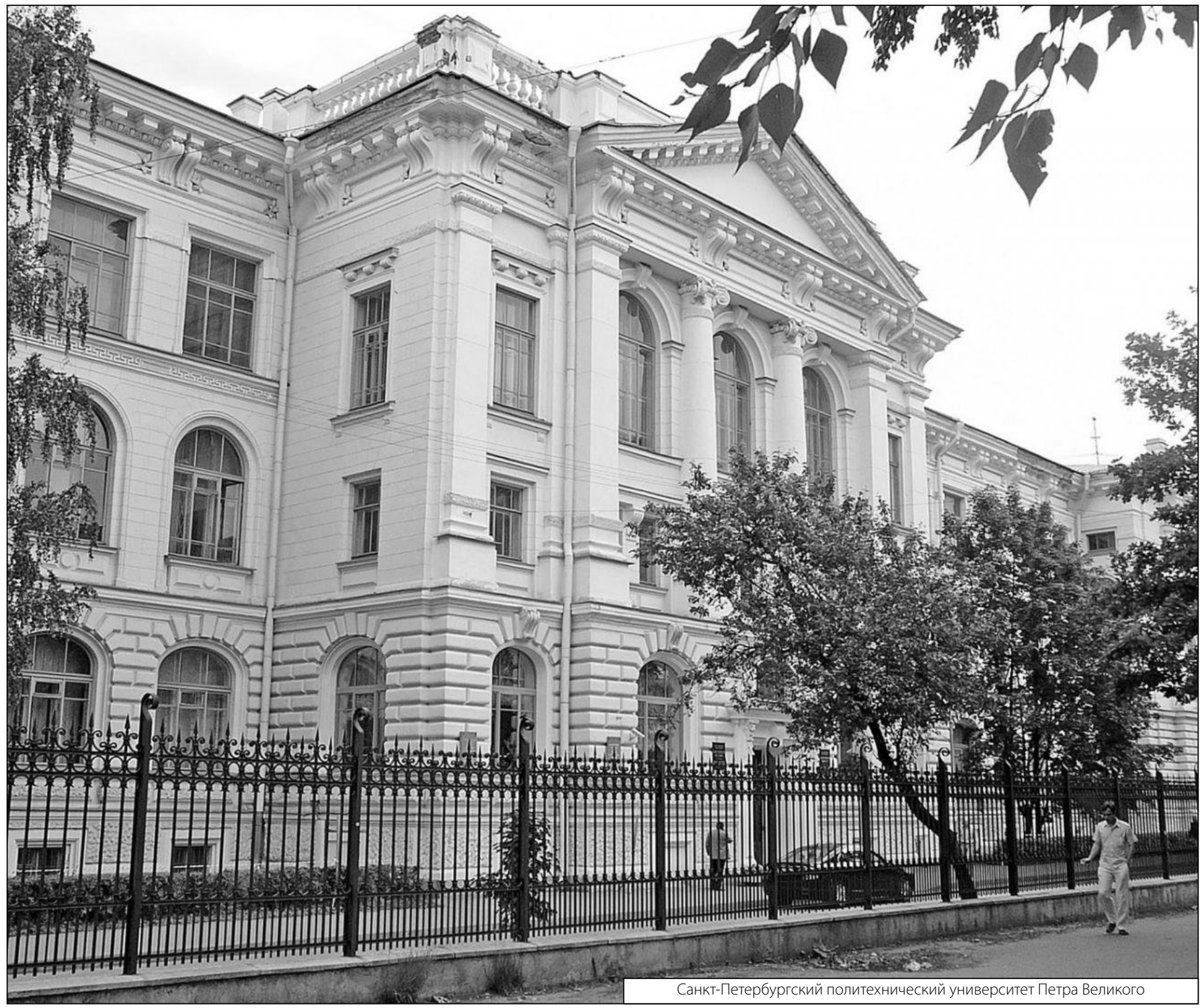

